Recepción: 20 / 04 / 2017

Aceptación: 20 / 05 / 2017

Publicación: 15 / 06 / 2017
Ciencias Económicas

Artículo de Investigación

\title{
Influencia de los patrimonios naturales mundiales en las economías locales
}

\author{
Influence of global natural heritage on local economies
}

\section{Influência do património natural mundial nas economias locais}

\author{
Pablo A. Vela-Riera ${ }^{\mathrm{I}}$ \\ pvela@utb.edu.ec \\ Zoila N. Merino-Acosta II \\ zmerino@utb.edu.ec \\ Maritza del Rocío Vela-Riera
ing-22@ hotmail.com \\ Maritza A. Rosero-Vela IV \\ maritza.rosero@hotmail.com
}

Correspondencia: pvela@utb.edu.ec

I. Facultad de Administración Finanzas e Informática; Magister en Educación con Mención en Educación Superior. Ingeniero Comercial Especializado en Recursos Humanos. Docente a tiempo completo en la Facultad de Administración, Finanzas e Informática (F.A.F.I.) de la Universidad Técnica de Babahoyo, Los Ríos, Ecuador.

II. Magister en Investigación de Mercados, Magister en Sistemas de Información Geográfica, Magister en Docencia y Currículo. Ingeniera en Estadística Informática. Docente a medio tiempo en FAFI, de la Universidad Técnica de Babahoyo, Los Ríos, Ecuador.

III. Magister en Educación, Ingeniería en Gestión Administrativa, Licenciada en Ciencias de la educación, Profesora de Segunda Enseñanza. Docente Evaluadora Universidad Católica Técnica Particular de Loja, Loja, Ecuador.

IV. Ingeniera en Administración de Empresas Turísticas y Hoteleras, Maestrante en Administración de Empresas Internacionales en Universidad Rey Juan Carlos de Madrid, Madrid, España. 


\section{Resumen}

El presente tema trata sobre el impacto de los acervos naturales del planeta en las finanzas de los grupos humanos localizados en su inmediatez. El objetivo del actual trabajo investigativo es analizar cómo inciden en las economías locales de las poblaciones circunscritas a los Patrimonios Mundiales Naturales (PMN) el grado de conservación o no de éstos sitios. La investigación se realizó bibliográficamente buscando los efectos económicos que sufren las poblaciones en relación a la afectación o cuidado de los PNM.

El presente tema se llevó a cabo acudiendo a varias fuentes de información científica y técnica que facilitaron el recabar datos de la situación actual y futura de los PNM y de las actividades económicas que se dan en estos sitios que marcan la calidad de vida de los habitantes de estos lugares sobresalientes. En conclusión, este estudio es un análisis del estado de los PNM y de las consecuencias en las rentas locales que se derivan de estas herencias naturales.

Palabras clave: Economías locales; influencia; patrimonio natural mundial. 


\section{Abstract}

The present theme is about the impact of the natural collections of the planet on the finances of human groups located in its immediacy. The objective of the current research work is to analyze how the conservation of these sites affects the local economies of the populations circumscribed to the World Natural Heritage (PMN). The research was done bibliographically looking for the economic effects that the populations suffer in relation to the affectation or care of the PNM.

The present theme was carried out by various sources of scientific and technical information that facilitated the collection of data on the current and future status of the NMPs and the economic activities that occur in these sites that mark the quality of life of the inhabitants Of these outstanding places. In conclusion, this study is an analysis of the state of NMPs and the consequences on local incomes that derive from these natural inheritances.

Key words: Local economies; influence; world natural heritage. 


\section{Resumo}

O tema atual é sobre o impacto das coleções naturais do planeta sobre as finanças de grupos humanos localizados em sua imediação. O objetivo do trabalho de pesquisa atual é analisar como a conservação desses sites afeta as economias locais das populações circunscritas ao Patrimônio Natural Mundial (PMN). A pesquisa foi feita bibliograficamente buscando os efeitos econômicos que as populações sofrem em relação à afectação ou cuidado do PNM.

O tema atual foi realizado por várias fontes de informações científicas e técnicas que facilitaram a coleta de dados sobre o status atual e futuro dos NMPs e as atividades econômicas que ocorrem nesses sites que marcam a qualidade de vida dos habitantes. locais. Em conclusão, este estudo é uma análise do estado dos NMPs e as conseqüências sobre os rendimentos locais que derivam dessas heranças naturais.

Palavras chave: Economias locais; influência; herança natural mundial. 


\section{Introducción.}

Se entiende por "patrimonio natural" a:

Los monumentos naturales constituidos por formaciones físicas y biológicas o por grupos de esas formaciones que tengan un valor universal excepcional desde el punto de vista estético o científico. (Pastor Alfonso, 2003)

Las formaciones geológicas y fisiográficas y las zonas estrictamente delimitadas que constituyan el hábitat de especies, animal y vegetal, amenazadas, que tengan un valor universal excepcional desde el punto de vista estético o científico. (Cluzeau, 2000)

Los lugares naturales o las zonas naturales estrictamente delimitadas, que tengan un valor universal excepcional desde el punto de vista de la ciencia, de la conservación o de la belleza natural. (UNESCO, 1972).

Los sitios de herencia natural del planeta “están cada vez más amenazados de destrucción, no sólo por las causas tradicionales de deterioro sino también por la evolución de la vida social y económica que las agrava con fenómenos de alteración o de destrucción aún más temibles”. (UNESCO, 1972)

El presente tema es de gran importancia en el presente siglo ya que "las emisiones de dióxido de carbono a nivel mundial se han incrementado en más de 50\% desde 1990. Enfrentar el aumento sin freno de las emisiones de gases de efecto invernadero y los posibles impactos resultantes del cambio climático, tales como la alteración de ecosistemas, las condiciones climáticas extremas y los riesgos a la sociedad, continúa siendo un desafío urgente y crítico para la comunidad global”. (ONU, 2015). 
La trascendencia del presente trabajo investigativo se refleja en los imprevisibles efectos desbastadores que pueden resultar del daño de los PMN. "Se estima que cerca de un cincuenta por ciento (50\%) de éstos están en muy grave peligro. Son sitios que están distribuidos en noventa y seis (96) países en el mundo. Donde once millones de personas $\left(11^{\prime} 000.000\right)$ dependen de éstos espacios".

El direccionamiento de la eficacia del presente tema y su aplicación no es sólo que se sepa de las amenazas a los PMN y la afectación negativa en statuo quo de sus pobladores, sino tomar conciencia de lo importante que es tomar urgentes medidas preventivas para frenar o por lo menos minimizar los daños que se está ocasionando a estos espacios y, asimismo, tomar decisiones inmediatas para su conservación. "La apuesta es, que se hace con lo que se tiene para mejorar el nivel de vida y la conservación del ecosistema, de manera que permita una mejor convivencia humana, un mejor entendimiento entre el planeta y quienes lo habitan". (La Filososfía de las empresas y su Responsabilidad Social, 2016).

Por lo tanto, la importancia de este tema es la creación de un nuevo paradigma, de "un cambio a fuentes de energía sostenibles y renovables, que no se basen en la extracción" (AMÉRICA E. , 2016), donde los gobiernos de todos los países en el mundo, especialmente los que más afectan a éstos patrimonios, asuman la responsabilidad ética e histórica e implementen de manera prioritaria políticas de estado a largo plazo que coadyuven a la minimización y, en su mejor caso, a la extinción de las amenazas que pesan sobre los PMN, asimismo, que las grandes, medianas y pequeñas industrias y empresas apliquen normas de cuidado ambiental en sus procesos. Los ciudadanos en general y, especialmente los habitantes del entorno de estos espacios y de cuya conservación dependen sus ingresos y su sobrevivencia, deben asumir la responsabilidad moral y social para que sean un aporte al cuidado del ecosistema aplicando 
reciclajes y normas de custodia ambiental en sus casas, en sus hogares y en el entorno que los rodea.

La esencia del cuidado ambiental que ejerzan los gobernantes y sus ciudadanos nace del seno familiar donde se adquiere, se vive y se convive con principios y valores que deben estar ligados a la atención y el cuidado de la familia y el entorno en que se vive. Nuestro país el Ecuador y su nación deben ser un referente de aplicación de principios y valores ya que "La naturaleza o Pacha Mama, donde se reproduce y realiza la vida, tiene derecho a que se respete integralmente su existencia y el mantenimiento y regeneración de sus ciclos vitales, estructura, funciones y procesos evolutivos" (Asamblea Constituyente, 2007)

\section{Problemática.}

Hay reservas gravemente afectadas como “el Sistema de Reservas de la Barrera del Arrecife de Bélice, más de la mitad de su población se sostiene con ingresos generados por la pesca y el turismo. Desde 1998, la construcción insostenible en la costa, la tala a gran escala de los manglares, la escorrentía" (corriente de agua que se vierte al rebasar un depósito o cauce. Aliviadero) "resultante de la agricultura y las exploraciones petroleras han dañado $40 \%$ de este lugar. El Arrecife de Bélice es el hogar de tres tipos de tortugas marinas, incluidas las verdes que están en peligro crítico".

Como antecedente del desgaste de los PNM se tiene la erosión natural causada por el viento y factores térmicos normales. Sin embargo, no es uno sino varios los graves problemas que afectan peligrosamente a los PNM acelerando meteóricamente su deterioro, entre ellos los más desbastadores son: la actividad industrial dañina, la amenaza no extractiva, el cambio climático y la convivencia humana no regulada. 
En lo que respecta a nuestro país, "la diversidad en el Ecuador está constantemente amenazada por los problemas ambientales que se derivan de la globalización y la tecnología: la deforestación, la contaminación del agua y del suelo".

El objetivo de este trabajo es predecir la afectación de la economía de la población que vive en los sitios considerados Patrimonios Naturales Mundiales (PNM) debido a su conservación o a un apresurado deterioro de éstos. "Según las proyecciones, los impactos sobre los sistemas naturales y humanos abarcarán todo el mundo, con efectos variables de región en región. Incluyen ecosistemas y hábitats alterados, impactos perjudiciales en la agricultura que podrían llevar a la escasez alimentaria”. (ONU, 2015).

Para ello describiremos los sitios de Latinoamérica y Centroamérica más afectados, las amenazas que aceleran su deterioro y las consecuencias económicas que se derivan de ello. Además, se ejemplarizará una propuesta de principios de protección para los PNM.

\section{Hipótesis.}

Si no hay una adecuada conservación de los PNM no hay desarrollo económico sustentable de las economías locales.

"Haití es un ejemplo abrumador del "circulo vicioso" que forman la extrema pobreza y la deterioración del medio ambiente. Gran parte de la pobreza y de los padecimientos del pueblo haitiano proceden de la pérdida de sus bosques”. (PNUMA/Topham, 2008)

\section{Metodología.}

Para el cabal cumplimiento de este trabajo se utilizaron los métodos analítico y sintético.

Se analizaron las actividades que inciden en la conservación y en el deterioro acelerado de los PNM así como los efectos positivos o nocivos sobre las economías locales dependiendo 
del caso, se apeló a varios pronunciamientos, con relación al tema, de notables autores y de avalados organismos con las consiguientes conclusiones de las investigaciones y consultas brindando resultados, conclusiones y recomendaciones integrados.

Los enfoques empleados, con respecto al tema tratado, son la sobrevivencia de los PNM y sus habitantes circunscritos y la decadencia o desaparición de los mismos junto con sus asentamientos humanos.

\section{Temática.}

1. Los Patrimonios Naturales Mundiales (PNM)

2. Aportación de los PNM al planeta y a la sociedad

3. Amenazas a los sitios considerados Patrimonio Natural Mundial

4. Sitios de Latinoamérica y Centroamérica amenazados

5. Principios de protección

6. Caso de éxito

\section{Desarrollo de la temática.}

\section{Los Patrimonios Naturales Mundiales (PNM)}

Junto con el pronunciamiento de la UNESCO (Organización de las Naciones Unidas para la Educación, la Ciencia y la Cultura) sobre los PNM revisemos otros criterios el patrimonio es potencialmente el recurso primario de una industria potente, la industria del turismo, y como tal debe generar por encima de todo renta. Es obvio que existe una dimensión del patrimonio que 
tiene que ver con la economía y la generación de recursos económicos, pero esta dimensión no debe tener un carácter predominante. (Ballart Hernández \& Treserras, 2005)

Se considera que el concepto de capital no puede ser aplicado a los elementos de la Naturaleza. Con ese fin se debe apelar al concepto de patrimonio. Recordemos que el término patrimonio hace referencia tanto a los bienes que se heredan, como a los legados que se dejan a los hijos. Asimismo, el concepto alude a bienes que antes estaban recubiertos de valores espirituales y hoy son capitalizados. (Gudynas , 2000)

Refiriéndonos al artículo publicado en Guayaquil-Ecuador por Diario el Universo con respecto a los PNM tenemos:

Aportación de los PNM al planeta y a la sociedad

\section{Suministran agua}

Apoyo al desarrollo sostenible y a la conservación de los ecosistemas naturales

Más de la mitad del PNM ayudan a estabilizar el suelo previniendo inundaciones.

Reservorios de carbono (con el oxígeno forman el dióxido de carbono vital para el crecimiento de las plantas, también forman los ácidos grasos, esenciales para la vida, y los ésteres que dan sabor a las frutas; también es factor, a través del ciclo carbono-nitrógeno, de parte de la energía producida por el sol).

Contribuyen al desarrollo de la economía de los países a través del turismo, la recreación y la exportación de productos.

Contribuyen al alivio de la pobreza: más del $90 \%$ de los sitios considerados PNM generan empleo. 
Los pobladores de estos sitios sobresalientes sobreviven y generan ingresos gracias a procesos productivos como:

La pesca

El turismo comunitario: guianza, servicios de bares y restaurantes, servicios de hospedaje, alquiler de animales y vehículos para paseos, venta de artesanías y productos propios del lugar, etc.

Amenazas a los sitios considerados patrimonios naturales del mundo

Actividad industrial dañina

- Sobrepesca

- Exploración de gas y petróleo

- Minería

- Uso insostenible del agua

- Tala ilegal

Amenaza no extractiva

- Uso no sostenible del agua

- Construcción de infraestructura vial. Las carreteras pueden dar paso a la explotación de la madera o tala del bosque.

\section{El cambio climático}

Convivencia humana en sitios sin contemplar regulaciones ambientales. (AMÉRICA E. ).

\section{Inundaciones}


Sitios de Latinoamérica y Centroamérica amenazados. (UNIVERSO, 2016).

\begin{tabular}{|c|c|c|}
\hline No. & Países & Sitios más afectados \\
\hline 1 & Argentina & 3 \\
\hline 2 & México & 3 \\
\hline 3 & Perú & 3 \\
\hline 4 & Bolivia & 3 \\
\hline 5 & Costa Rica & 3 \\
\hline 6 & Panamá & 1 \\
\hline 7 & Ecuador & 1 \\
\hline
\end{tabular}

\section{Tabla 1.- Sitios afectados más afectados en Latinoamérica y Centroamérica, de mayor a menor.}

Fuente: Elaboración propia en base a información de El Universo (2016)

\section{Sitio de Ecuador amenazado}

De Ecuador, los Parques Nacionales Galápagos y Sangay son los Patrimonios Mundiales de la UNESCO. Este último designado Patrimonio en 1983, ya fue declarado en amenaza en 1992. El Parque Nacional Sangay está conformado por 517.765 hectáreas que albergan a 343 especies de aves, 100 de mamíferos, 25 anfibios y reptiles. La carretera La Riobamba-Macas, una vía de acercamiento al sector, el Ministerio del Ambiente y la Unidad de Protección del Medio Ambiente de la Policía Nacional han desplegado operativos en los que han decomisado madera nativa ilegal, según reportes que constan en la página web del MAE. Aunque parezca increíble, un simple error humano puede potencialmente eliminar a toda una especie; es una idea desalentadora, pero ya ha sucedido: el derrame de petróleo del 2001 mató al 60\% de las iguanas marinas de las Galápagos, una especie endémicadelasislas. 


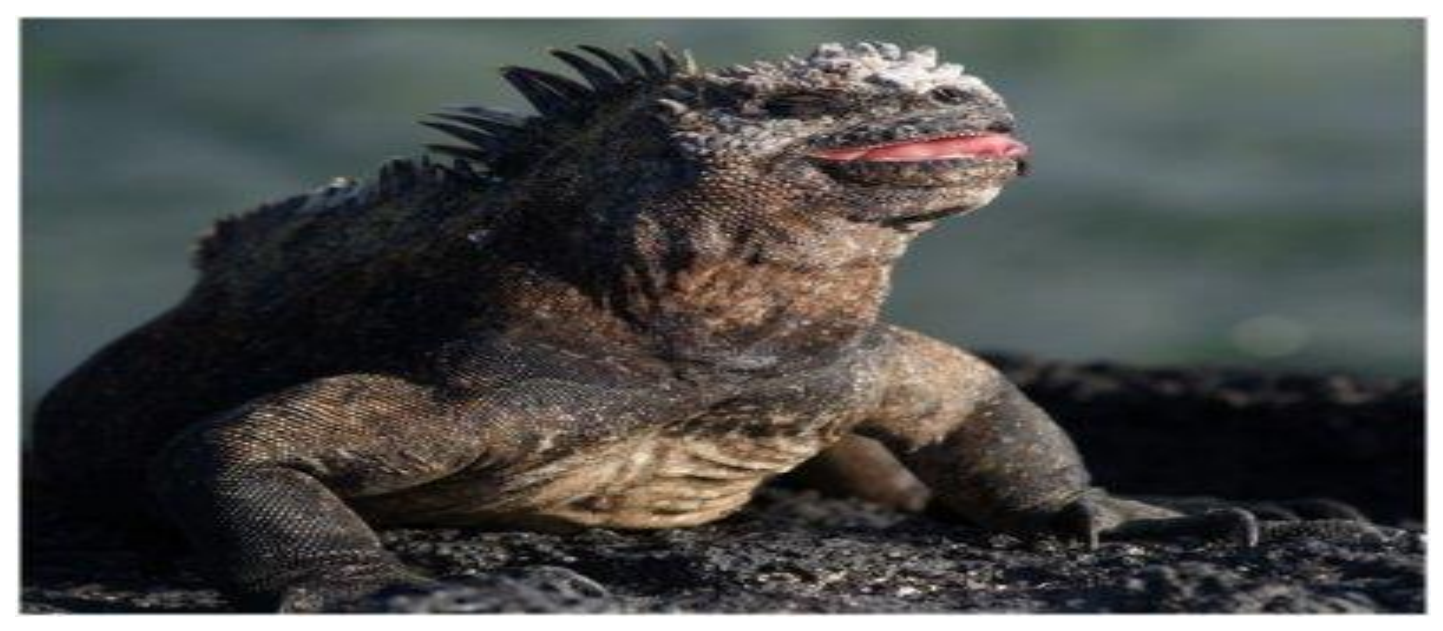

Figura 1.- Iguana de las Islas Galápagos.

Fuente: (AMÉRICA E. )

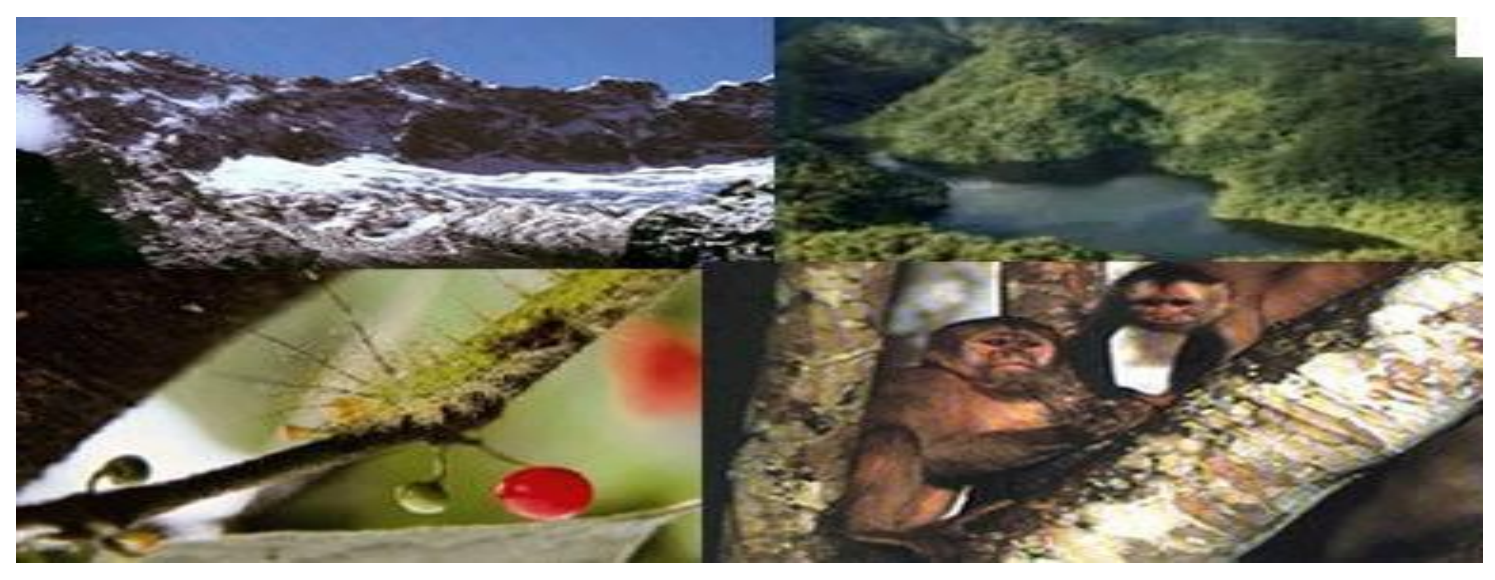

Figura 2.- Parque Nacional Sangay

Fuente: (ecuador-turistico.com, 2012) 


\section{Principios de protección}

\section{Primero}

Evaluar periódicamente el valor directo, indirecto y de no utilización de los sitios de Patrimonio Mundial

Segundo

Evaluar si al hacer inversiones en actividades se pudieran afectar los sitios del PNM y a las personas que dependen de ellos.

Tercero

Involucrar a los diversos actores, sobre todo a las comunidades locales, en el cuidadoso manejo de los sitios

\section{Cuarto}

Políticas de procesos inclusivo y transparente.

\section{Quinto}

Aplicar el principio del cumplimiento de las normas que protegen a los PNM.

\section{Caso de éxito}

El Parque Natural Arrecife de Tubataha, en Filipinas, dejó de verse afectado por la pesca excesiva e insostenible luego de que su gobierno declarara al área que alberga más de 600 especies como zona de exclusión pesquera y redobló sus esfuerzos para sancionar la pesca ilegal. Esfuerzos que han derivado en que se cuadripliquen los inventarios de peces. 


\section{Discusión de resultados.}

Culminado el presente trabajo se puede evaluar que los PNM afectan directamente a la economía de las poblaciones ubicadas en estos sitios. De acuerdo a los enfoques empleados en el presente trabajo se arrojan los siguientes resultados:

Si la conservación de los PNM es la adecuada minimizando su deterioro al mínimo:

a) Los pobladores de estos lugares podrán seguir generando ingresos por la pesca regulada, incluso, no faltará este medio de subsistencia y de ingresos por la misma aplicación de normas que impiden la pesca indiscriminada atentando a su desaparición.

b) Los pobladores de estos lugares podrán seguir generando ingresos por el turismo, por servicios de guianza, por los servicios de restaurantes, por los servicios de hospedaje, etc.

c) Se verán beneficiados indirectamente por las inversiones que se realizan para la conservación del ecosistema.

Si la conservación de los PNM no es la adecuada aumentando su deterioro natural:

a) Los pobladores de estos lugares no podrán seguir generando ingresos porque la pesca es indiscriminada o porque ésta no es regulada o no se cumplen con las regulaciones, incluso, faltará este medio de subsistencia y, por lo tanto, ingresos por la merma de existencias de peces.

b) Los pobladores de estos lugares no podrán seguir generando rentas por el turismo y venta de productos y servicios colaterales.

c) Se verán beneficiados indirectamente por la merma o cese de inversiones que se realizan para la conservación del ecosistema. 


\section{Conclusiones.}

Las conclusiones que se derivan del presente trabajo investigativo son las siguientes:

Los PNM influyen directamente en las economías locales al incidir en su nivel de ingresos

La adecuada conservación de los PNM permite que sus pobladores puedan seguir ofertando servicios y bienes que les permite tener finanzas sustentables.

El deterioro acelerado de los PNM causan el colapso de los recursos naturales en los sitios, merman las especies acuáticas, decae el atractivo turístico, y quienes visitan estos lugares se desaniman y se dirigen a otros lugares lo que afecta la demanda ocasionando disminución de las rentas de sus pobladores.

\section{Recomendaciones.}

Los PNM son legados que la misma naturaleza pródiga brinda a quienes viven en el planeta dándoles los recursos necesarios para su sobrevivencia, depende de los seres humanos, quienes habitan este este mundo, reconocer su valía y realizar todo lo posible, aplicando normas y regulaciones junto con modelos de desarrollo, para la conservación de estos sitios sobresalientes según el concepto mencionado de la UNESCO.

Con respecto al Ecuador "tiene muchos recursos tales como el sol y el agua, que se pueden utilizar para generar energía de manera más ecológica y sostenible en comparación con la extracción y el consumo de combustibles fósiles. El uso de sistemas de paneles solares, la energía hidroeléctrica, los mecanismos para capturar la energía del viento y las fuentes de energía geotérmica, permitirían al Ecuador avanzar hacia un futuro más sostenible, pero son 
necesarias la acción humana y la responsabilidad para que se produzca cualquier cambio significativo.

\section{Bibliografía.}

Ecuador-turistico.com. (Abril de 2012). Obtenido de http://www.ecuador-turistico.com/2012/04/parquenacional-sangay-sitios-y-lugares.html

AMÉRICA, E. (26 de 02 de 2016). ecoticias.com. Obtenido de http://www.ecoticias.com/ecoamerica/124656/Los-mas-acuciantes-problemas-ambientales-en Ecuador

AMÉRICA, E. (s.f.). www.ecoticias.com. Obtenido de http://www.ecoticias.com/ecoamerica/124656/Los-mas-acuciantes-problemas-ambientales-en Ecuador

Asamblea Constituyente. (2007). Constitución Nacional. Montecristi.

Ballart Hernández, J., \& Treserras, J. (2005). Gestión del Patrimonio Cultural (2a ed. ed.). Barcelona.

Cluzeau, C. (2000). Origet du. Le tourisme culturel. Paris: Puf.

Gudynas , E. (2000). Los límites de la sustentabilidad débil, y el tránsito desde el capital natural al patrimonio ecológico. Educación, Participación y Ambiente, MARN, 4(11), 7-11.

ONU. (2015). OBJETIVOS DE DESARROLLO DEL MILENIO. NEW YORK: ONU.

Pastor Alfonso, M. (2003). El patrimonio cultural como opción turística. Horizontes Antropológicos, $9(20)$.

PNUMA/Topham. (2008). La economia de los ecosistemas y la biodiversidad. Belgium: Comunidades Europeas.

UNESCO. (1972). Convención para la protección del patrimonio mundial, cultural y natural. (pág. 16). Paris: WWF.

UNIVERSO, D. E. (2016). ECOLOGÍA. GUAYAQUIL: DIARIO EL UNIVERSO.

Vela Riera, P. A., \& Izquierdo Morán, J. (2016). La Filososfía de las empresas y su Responsabilidad Social. (J. M. Arístega, Ed.) Revista Científica FINYTEC de la UTB, 1(1), 81. 